\title{
Beyond the bench: skills needed for success in the pharmaceutical industry
}

\author{
Wendy Saffell-Clemmer ${ }^{1}$
}

Accepted: 18 March 2021 / Published online: 30 March 2021

(C) Springer-Verlag GmbH Germany, part of Springer Nature 2021

As a scientific leader at a global healthcare company, I have often been asked to provide insight to Biotechnology and Chemistry programs on essential skills for graduates. Coursework in mathematics and fundamental science as well as familiarity with basic laboratory skills are the foundation on which other softer skills must be developed for a long-term career as a scientist in industry. While relevant laboratory course experience may help new graduates contribute immediately in their first positions, the pace of change in the way we work, the tools we use, and regulatory requirements require life-long skill development.

\section{Global teamwork and collaboration}

When most people imagine the role of analytical scientist, they picture a lone individual in the laboratory, in a lab coat and safety glasses working at a laboratory bench performing sample dilutions or in front of a computer controlling complex instrumentation. While laboratory activities are critical, the time physically spent in the laboratory tends to be a small portion relative to time spent on study design, data analysis, documentation, and communication. In regulated industries such as pharmaceuticals, current Good Manufacturing Practices (cGMPs) extend to development laboratories, increasing requirements for documentation of study design, execution, and results. Further in contrast to the image of the solitary scientist, almost all product development and product quality work is performed by a team and collaboration is a key skill for all employees. More often than not, project teams are not physically co-located, and instead can span multiple countries and time zones. As a US-based team member in a global

Wendy Saffell-Clemmer

wendy_saffell_clemmer@baxter.com

1 Baxter Healthcare Corporation, 927 S. Curry Pike, Bloomington, IN 47401, USA company, I circle the globe daily, working with colleagues in India and Europe in the morning, the USA throughout the day, and ending with meetings with team members in China or India in the evening. The demands of global coordination require that team members share information via electronic systems to allow accessibility by all locations at all hours. Additionally, team members must communicate timelines, results and decisions quickly, and often asynchronously, so that work can continue around the globe. Global information sharing and communication require strong organization and the use of new cloud-based systems such as Electronic Laboratory Notebooks (ELN), data management tools (SharePoint and Tableau Software), and communication tools such as Microsoft Teams ${ }^{\circledR}$. Analytical scientists must be able to communicate results to project teams and stakeholders clearly through both formal reports and presentations and informally through virtual team meetings and electronic communication tools.

COVID-19 has reduced the amount of in-person collaboration and training that could be done in the laboratory and increased the reliance on electronic tools used for global communication. In the past, electronic tools were used solely for communication in situations when colleagues were physically distant, and travel was prohibitively expensive. As we responded to the pandemic by reducing laboratory capacity and mandating that employees distance from one another, in-person training became challenging for team members at the same location and electronic tools originally intended for global communication were used by colleagues in the same facility. Examples from my teams included making simple videos to train team members on a specific sampling technique and using screen sharing using video conferencing tools to train new scientists how to navigate complex instrument control software.

The pandemic has presented challenges to educators attempting to lead laboratory courses safely and some have employed solutions such as having lab partners work in shifts. Rather than viewing this as a setback, instead educators 
should understand that the requirement to communicate progress to a team member via written documentation and electronic tools is preparation for today's global workplace.

\section{An inclusive workplace drives productivity}

In addition to strong written, verbal, and communication skills, scientists must be trained to work inclusively across cultures to collaborate with global project teams, external service partners, and internationally based raw material suppliers. The correlation of team diversity with positive outcomes in improved innovation has been widely reported [1]. Healthcare is provided differently across global markets, not limited to diversity in location of use, preferences for delivery system, challenges in distribution and storage. Diverse individuals help teams understand their community's specific preferences and the resulting products can better meet user needs across diverse markets. For a team to reap the benefits of diverse thoughts, it is critical that team members have the skill to respectfully listen and respond in a manner that cultivates a safe space for ideas and innovation. Valuing differences is critical to creating a productive and inclusive working environment and many large healthcare companies provide opportunities for employees to expand their cultural awareness and cross-cultural communication skills. Baxter employees are encouraged to complete Cultural Navigator ${ }^{\circledR}$ [2] training to understand their own cultural preferences and learn how these compare to the norms in other countries. Baxter also sponsors internal Business Resource Groups (BRGs), which provide forums to enhance engagement and increase the impact in employee communities such as the Baxter Black Alliance, the Asian Leadership Network, and Latinos @ Baxter. The BRGs provide employees with opportunities and programs to enhance their cultural awareness.

Educators can foster an inclusive environment through teamwork in which different ideas for project execution must be discussed and evaluated. The assignment of diverse teams, rather than allowing students to self-select from their friendgroup, will more closely mimic the today's business reality.

\section{Continuing education is essential}

Rapid changes in product types, scientific requirements, and regulatory expectations require that today's analytical chemist continuously develop their skills and knowledge through courses and seminars as well as self-learning and completion of assignments which require the team member to stretch their skills or knowledge in new areas or directions. Development of learning agility, sometimes defined as "knowing what to do when you don't know what to do," can lead to employees who learn from challenging assignments and can translate their knowledge from their own area of expertise across functions and businesses. The role of today's analytical scientist is no longer limited to sample analysis and data generation. In collaborative project teams, the analytical scientist is expected to contribute to product development, design characterization and analytical plans, and investigate impurities or other unexpected outcomes. The analytical scientist on a pharmaceutical development team must understand and anticipate changes in the global regulatory environment ensuring that characterization and testing plans are designed to justify the safety and quality of the new product even when standards are evolving and ambiguous.

Pharmaceutical development is cross-disciplinary and depending on project needs. Therefore, the analytical scientist must find ways to educate themselves across functions to bridge these gaps. For example, validation of cleanliness of equipment through sampling and testing is an important aspect of pharmaceutical manufacturing. Analytical test methods must be developed and validated to test rinse and swab samples from equipment. However, to develop and validate the test method, the analytical scientist must be aware of the manufacturing equipment cleaning procedures, materials, cleaning agents, and sampling methods employed at the manufacturing facility so that surface recovery and method performance in the sample matrix can be demonstrated. The analytical level of that test method will be set by the allowable carryover limit determined by the product toxicity, expressed as its permissible daily exposure (PDE), as well as the sampling volumes used at the manufacturing facility. Analytical scientists must communicate with toxicologists, manufacturing equipment experts, and manufacturing team members. Another example of a cross-disciplinary project is the determination of the safety of pharmaceutical product containers through testing to understand the potential for compounds to be leached from the container into the product during its storage period. Extractable and leachable studies are typically performed using complex analytical equipment such as gas chromatography-mass spectrometry (GC-MS) and liquid chromatography-mass spectrometry (LC-MS) and inductively coupled plasma-mass spectrometry (ICP-MS) to detect potential leachables at extremely low analytical levels. The analytical evaluation levels of these studies are dictated by the maximum daily dose of the product requiring knowledge of the medical use of the pharmaceutical product. Any leachables detected above a safety concern threshold must be identified and assessed by toxicologists. The study director for an extractables and leachables assessment must compile input from materials science, medical, toxicology, and regulatory team members to design a testing strategy and prepare a narrative explaining how the testing results and toxicological assessment demonstrate the safety of the drug product throughout its storage life.

Educators can increase learning agility in students by including more ambiguity in laboratory procedures, such as 
allowing students to select what analytical test methods might be most suitable for use in analysis. Additionally, laboratory courses which bridge disciplines or require students to investigate other fields of study to locate required inputs would add real-world context to laboratory experiments.

\section{Feedback is a gift}

One of the most effective ways for scientists to develop their skills in communication, teamwork and collaboration, and learning is by listening to and acting on constructive feedback. Giving and receiving feedback is not always something that comes easily and is itself a skill to be developed. Feedback is a gift and provides both an opportunity to recognize and build on success as well as highlight areas for improvement. Constructive feedback can be used by managers to help employees understand where they are failing to meet expectations and provide areas for improvement. Equally valuable, feedback from peers and project team members can help team members develop self-awareness around failures to communicate or situations that could be handled differently in the future. It takes courage to give constructive feedback and practice is needed to ensure that it is delivered in such a way that it is specific and focused on improvement. Listening to feedback and separating emotional reactions from an understanding of the substance is also a skill which develops with use.

Educators can incorporate practice in giving and receiving feedback through a "lessons learned" session at the conclusion of a group project to identify areas of improvement or by requesting students provide constructive feedback following presentations. Students can also act as peer reviewers for student laboratory reports.

\section{Conclusion}

Education does not stop at graduation and the most successful students will be those equipped to build on the foundation of scientific knowledge gained through coursework. Providing students with opportunities to work in diverse teams to develop their collaboration and communication skills will prepare them to develop products that will meet the user needs of patients globally. Assignments which challenge and build confidence in learning agility, the ability to manage ambiguity, and the ability to bring cross-discipline information together will prepare students to execute projects as part of a multi-functional team. Science and mathematics are the foundation of the preparation of today's analytical chemist. The soft skills of communication and learning agility will prepare today's graduates for life-long skill development ensuring they will adapt to new ways of working and new challenges in healthcare.

\section{Declarations}

Conflict of interest The author declares no competing interests.

\section{References}

1. Freeman RB, Huang W. Collaborating with people like me: ethnic co-authorship within the U.S. J Labor Econ, Special Issue on High Skill Immigration [Internet]. 2015;33 (3) :S289-S318.

2. https://www.culturalnavigator.com/CN7/login.aspx (accessed March 17, 2021).

Publisher's note Springer Nature remains neutral with regard to jurisdictional claims in published maps and institutional affiliations.

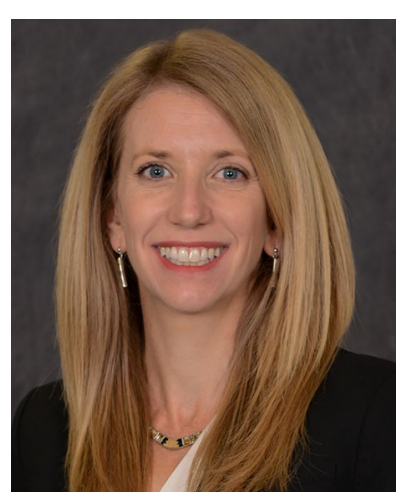

Wendy Saffell-Clemmer is Lead Scientist and Sr. Director of R\&D in the Global Pharmaceutical business of Baxter Healthcare. She leads teams based in the USA and India developing injectable drug products including formulation development and analytical development as well as characterization of containerproduct interactions. Her main area of interest is biologic products such as peptides, proteins, monoclonal antibodies, and antibody drug conjugates. 